\title{
INCIDENCIA DE FALLA DE CATÉTERES DE NERVIO PERIFÉRICO CONTINUO PARA ANALGESIA POSTOPERATORIA EN CLÍNICA SANTA MARÍA ENTRE LOS A NOS 2014-2016
}

Fernanda Bastías Moraga ${ }^{1}$, Constanza Manzano Pérez ${ }^{1}$, Carlos Yarur Spencer ${ }^{1}$, María Ignacia Troncoso Hugot ${ }^{1}$, Lorena Pinto Ramírez ${ }^{1}$, Enrique Moreno Cantariño ${ }^{1}$

1 Clínica Santa María.

Introducción: La analgesia otorgada a través de bloqueo de nervio periférico continuo es una herramienta eficaz y segura para el manejo del dolor postoperatorio, reduciendo requerimientos de medicamentos analgésicos y los efectos secundarios asociados a estos ${ }^{1}$. Sin embargo, es una técnica no exenta de falla, con incidencia reportada de $4-15 \%{ }^{2}$.

Objetivo General: Determinar la incidencia de falla de catéteres continuos de nervio periférico para analgesia postoperatoria en Clínica Santa María entre los años 2014-2016.

Material y Métodos: Tras la aprobación del comité de ética de Clínica Santa María, se analizaron en forma retrospectiva los datos de catéteres para bloqueo continuo de nervio periférico (BCNP) instalados durante el período de julio de 2014 hasta septiembre de 2016. Se registraron datos demográficos (sexo, edad), localización del catéter, EVA y falla de catéter, definida como cualquier evento que altere el funcionamiento de éste (ej: retiro accidental, desplazamiento, obstrucción del catéter), impidiendo una analgesia satisfactoria. Se describieron las causas de falla, se calculó el porcentaje de falla por tipo de catéter y la tasa de falla anual global.

Resultados: Durante el periodo analizado, se instalaron 751 BCNP: $513(68,3 \%)$ en extremidad superior, $225(29,96 \%)$ en extremidad inferior y 13 en tronco $(1,73 \%)$. De los catéteres instalados, en 63 pacientes se discontinuó su uso; de estos, 26 corresponden a retiro voluntario por solicitud de paciente o médico tratante y sólo en los 37 pacientes restantes se detectó fracaso de la técnica analgésica, lo que corresponde a una incidencia de falla global de $4,92 \%$. La incidencia por tipo de catéter se detalla en tabla 1 . Respecto a causas de falla, el $62,2 \%$ se debió a retiro accidental del catéter y un $37,8 \%$ a disfunción de este, explicada por desplazamiento $(24,3 \%)$, filtración $(8,1 \%)$, obstrucción y corte $(2,7 \%$ cada uno). En relación a complicaciones de la técnica, se reportaron 3 eventos adversos: 2 pacientes con parálisis frénica sintomática y 1 paciente con Síndrome de Claude Bernard Horner, que no implicaron retiro de catéter.

Conclusiones: La analgesia regional continua es una práctica habitual en clínica Santa María.

Tabla 1. Falla de catéter según sitio de inserción mas común

\begin{tabular}{|c|c|c|c|c|}
\hline Posición Catéter & $\mathrm{N}^{\circ}$ Catéter & $\% *$ & $\mathrm{~N}^{\mathrm{o}}$ Falla & $\% *$ \\
\hline Interescalénico & 362 & $48,20 \%$ & 24 & $3,19 \%$ \\
\hline Infraclavicular & 54 & $7,20 \%$ & 1 & $0,13 \%$ \\
\hline Retroclavicular & 85 & $11,30 \%$ & 0 & $0 \%$ \\
\hline Ciático Popliteo & 26 & $3,50 \%$ & 3 & $0,40 \%$ \\
\hline Ciático Vía anterior & 106 & $14,10 \%$ & 2 & $0,30 \%$ \\
\hline Canal de los Aductores & 60 & $8 \%$ & 4 & $0,50 \%$ \\
\hline Bloqueo Paravertebral & 13 & $1,73 \%$ & 1 & $0,13 \%$ \\
\hline
\end{tabular}

*Datos estadisticos extraidos del total de catéteres estudiados 
La incidencia de falla en nuestro centro está dentro de los rangos reportados en la literatura. El sitio de inserción con mayor porcentaje de falla de catéter corresponde al abordaje interescalénico, siendo el retiro accidental la causa más frecuente. Por esto creemos necesario optimizar y estandarizar medidas de fijación de catéteres para disminuir la falla por esta causal. Cabe destacar que existe un porcentaje de pacientes con retiro voluntario de catéteres funcionales, lo que nos insta a reforzar la educación del paciente y equipo tratante respecto al correcto uso de este dispositivo y sus beneficios asociados.

\section{Referencias}

1. Ilfeld, Anesthesia-analgesia 2011; 113;4 904925.

2. Zahab, J-Hand-Surg-Am, 2014; 39; 324-329. 